\title{
A Soft Strain Sensor Based on Ionic and Metal Liquids
}

\author{
Jean-Baptiste Chossat, Yong-Lae Park, Member, IEEE, Robert J. Wood, Member, IEEE and Vincent Duchaine
}

\begin{abstract}
A novel soft strain sensor capable of withstanding strains of up to $100 \%$ is described. The sensor is made of a hyperelastic silicone elastomer that contains embedded microchannels filled with conductive liquids. This is an effort of improving the previously reported soft sensors that uses a single liquid conductor. The proposed sensor employs a hybrid approach involving two liquid conductors: an ionic solution and an eutectic gallium-indium alloy. This hybrid method reduces the sensitivity to noise that may be caused by variations in electrical resistance of the wire interface and undesired stress applied to signal routing areas. The bridge between these two liquids is made conductive by doping the elastomer locally with nickel nanoparticles. The design, fabrication, and characterization of the sensor are presented.
\end{abstract}

Index Terms-Wearable sensors, microfluidics, strain measurement, ionic solution, eutectic gallium indium (eGaIn).

\section{INTRODUCTION}

$\mathbf{S}$ TRAIN gauges have been widely used as sensors in robotics [1]-[3] for their mechanical and electrical simplicity, compact form factors and cost effectiveness. They provide relatively high accuracy for measuring small strains. However, the measurement range of conventional strain gauges is limited by the material of the sensor, typically on the order of $1 \%$ or less, thus making them unsuitable for soft structures that may experience large deformations and strains.

Optical sensors [4]-[7] are used widely for measuring strain of greater magnitude but are often bulky and difficult to integrate into soft structures. Several researchers have proposed the use of silicone micro-composites [8] or more recently nano-composites [9], [10] to build piezo-resistive soft strain sensors. Unlike their metallic alternatives, sensors made of silicone can sustain very large elongations and typically have relatively high gauge factors [11] due to the bandgaps resulting from the change in the inter-atomic spacing. However, the latter characteristic makes their output highly nonlinear and thus difficult to predict [12]. Indeed, the change in the resistance of such materials is dominated by much more complex

Manuscript received March 4, 2013; revised April 22, 2013; accepted April 30, 2013. Date of publication May 17, 2013; date of current version August 6, 2013. The associate editor coordinating the review of this paper and approving it for publication was Prof. Boris Stoeber.

J.-B. Chossat and V. Duchaine are with Ecole de Technologie Supérieure, Montréal, Québec H3C 1K3, Canada (e-mail: jean-baptiste.chossat.1@ens.etsmtl.ca; vincent.duchaine@etsmtl.ca).

Y.-L. Park and R. J. Wood are with the School of Engineering and Applied Science and the Wyss Institute for Biologically Inspired Engineering, Harvard University, Cambridge, MA 02138 USA (e-mail: ylpark@wyss.harvard.edu; rjwood@eecs.harvard.edu).

Color versions of one or more of the figures in this paper are available online at http://ieeexplore.ieee.org.

Digital Object Identifier 10.1109/JSEN.2013.2263797 effects such as quantum tunneling [13], [14] rather than simple changes in geometry, making their responses nonlinear and often exhibiting hysteresis.

Highly stretchable sensors composed of a hyperelastic elastomer and embedded microchannels filled with a conductive liquid eutectic gallium-indium (eGaIn) have demonstrated relatively high accuracy and reliability for measuring large strains [15], [16]. Similar to conventional strain gauges, the change of the resistance of these soft sensors is a function only of the change in geometry, that is, the length and the crosssectional area of the microchannels. Their output is therefore nearly linear over a wide range of strain and hence easily predicted. These qualities make this sensor a good candidate for soft robotics applications. For example, Kramer [17] used this technology as a single axis curvature sensor to monitor the bending angle of a finger joint. However, there are certain limitations of the applicability of eGaIn for this purpose. Although its relative response in terms of resistance is reliable, the absolute difference between the resistance of the unbent and the fully bent sensor in [17] remains small, due to the high conductivity of eGaIn. As subsequently pointed out in [18], this low resistivity makes the sensor sensitive to electrical noise and variations in the interface resistance due to factors such as movement of connecting wires. Furthermore, since the entire sensor is wired with eGaIn, any external stimulus changing the geometry of a channel dedicated to signal routing could be interpreted as a strain.

The work presented here is a key aspect of a project [19] with the goal of creating an underactuated prosthetic hand [20] capable of restituting exteroception [21] as well as proprioception [22] to amputees. As part of the work on proprioception, we are currently designing a strain-sensing glove similar to that previously proposed [17] for the purpose of measuring the configuration of an artificial hand. The novelty of our approach is that we are using two conductive fluids differing in resistivity in order to decouple the signal due to hand movement, from noise due to undesired stress applied to signal routing channels.

One of the main challenges for soft sensors is routing the strain signal using soft wires. For example, soft wires can be composed of long channels filled with eGaIn. Stress applied to one of these channels would cause a resistance change and therefore an erroneous sensor reading.

Due to the unstructured nature of the environment in which a prosthetic hand will be used, it is obvious that soft wires located on the back of the hand will be subject to occasional stresses such as contact with an object. Therefore, in order to 

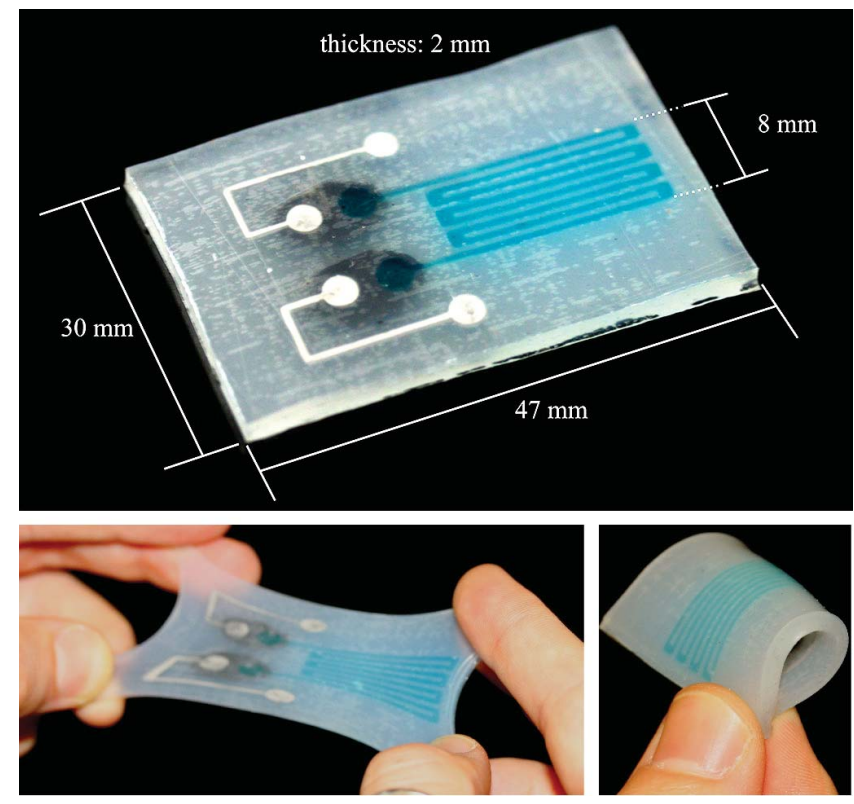

Fig. 1. Image of the hybrid soft strain sensor prototype.
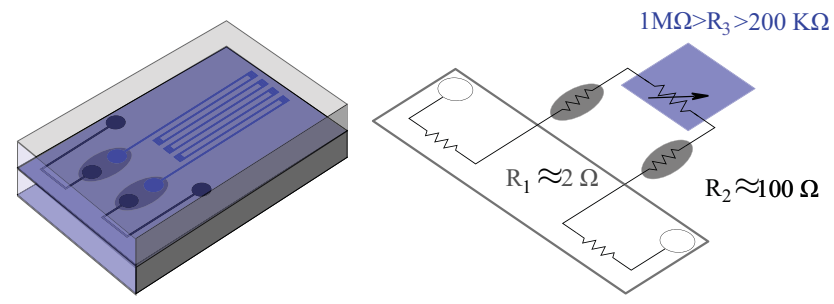

Fig. 2. Schematic of the sensor and its equivalent electrical circuit. $R_{1}, R_{2}$ and $R_{3}$ respectively being the eGaIn resistance, the silicon doped interfaces resistance, the ionic solution resistance.

make the whole glove sensitive only to strain applied at the desired measurement area, channels filled with ionic liquid are used as sensing elements while eGaIn channels are dedicated to routing the electrical signal as soft wires to a flexible printed circuit board (PCB) located at the wrist.

This hybrid approach is represented in the Figure 2, the superior conductivity of eGaIn and doped silicone are used respectively in channels and conductive interfaces, carrying electrical signals from the glove, while the resistance of the aqueous ionic solution is used as strain sensitive element. This results in a sensor with higher overall resistance and increased biocompatibility, potentially broadening its range of applications.

In this paper, we present the design of a single strainsensing element based on this hybrid approach (see Figure 1). Sections II and III present the design and assembly of the sensor, and section IV presents test results.

\section{DESIGN}

As mentioned in the introduction, our sensor uses two different conductive liquids. While this hybrid method brings us several key advantages, it also created two main design challenges that we had to overcome:
1) The two liquids are required to be kept separate in order to prevent them from being mixed. As a consequence, we created non-fluidic soft conductive interfaces that were embedded between the two different microchannels.

2) The use of an ionic solution introduces constraints in the design of the associated electrical circuit since we must avoid electrolysis of the solution and polarization of the electrodes. Thus, a custom-designed circuit applies a low voltage $\mathrm{AC}$ current centered at $0 \mathrm{~V}$ with a fixed frequency range is used to electrically interface with the sensor.

This section will therefore present more in detail the different elements of the sensor's design.

\section{A. Conductive Liquids}

Our goal is to produce a soft sensor that is sensitive to strain generated by the movements of a prosthetic hand, and relatively insensitive to strain or stress occurring elsewhere on the glove. This is achieved with higher resistivity in the sensing portion attached to the joints and a more conductive liquid in the signal-routing portions attached to the static surfaces of the glove.

1) Ionic Solution: The liquid used in the strain-sensitive portions of the sensor is a solution of sodium chloride $(\mathrm{NaCl})$ in water $\left(\mathrm{H}_{2} \mathrm{O}\right)$ containing glycerol (Atlas Laboratories). Glycerol was used for the increased viscosity of the solution $(1: 1$ water/glycerol by volume). $\mathrm{NaCl}$ was dissolved using a planetary mixer (Thinky ARE-310), which produces a centrifugal force of up to $400 \mathrm{~g}$ to ensure perfect dissolution. The solubility of $\mathrm{NaCl}$ in 1:1 water/glycerol being $21.8 \mathrm{~g}$ per $100 \mathrm{ml}$ at room temperature ${ }^{1}$, our solution contained $19 \mathrm{~g}$ of $\mathrm{NaCl}$ per $100 \mathrm{ml}$. With this concentration, no precipitate formed in the sensor even with pressure or temperature variations.

The use of Ionic solutions for sensing have been previously proposed. Cheung et al. have proposed a cylindrical strain sensor using a saline solution and reported the analysis of the impedance of the solutions over a very wide range of frequency and strain [23]. However, the sensor was considerably larger than in the present study and involved the use of rigid metal electrodes. These two characteristics would make this sensor very difficult to be integrated into a compact, portable and soft application such as a proprioceptive glove. Ionic liquids (IL) have also been used for monitoring fluid pressure in a microfluidic system in order to characterize flow conditions and liquid properties [24].

2) Eutectic Gallium-Indium: The second liquid used in our sensor is an eutectic metal alloy consisting of gallium (75\%) and indium (25\%) (eGaIn, Sigma-Aldrich). Maintaining its liquid state at room temperature $\left(15.7^{\circ} \mathrm{C}\right.$ melting point), this alloy and Galinstan [25], a similar alloy, are considered non-toxic alternatives to mercury [26]. With a resistivity of only a few $\mathrm{m} \Omega / \mathrm{cm}\left(29.4 \times 10^{-6} \Omega / \mathrm{cm}\right.$ [26] $)$, eGaIn has a conductivity close to that of copper. Both eGaIn and Galinstan

\footnotetext{
${ }^{1}$ The solubility of $\mathrm{NaCl}$ at room temperature is $35.7 \mathrm{~g}$ per $100 \mathrm{ml}$ in water and $7.8 \mathrm{~g}$ per $100 \mathrm{ml}$ in glycerol.
} 
have been used recently in flexible and stretchable electronics [27]-[30].

In our sensor, microchannels filled with eGaIn are used to supply electric currents to the strain-sensitive portion of the sensor and transmit the sensor signal. As previously demonstrated [23], the resistivity of the saline solution is much greater than that of eGaIn. Indeed, the conduction mechanism of saline solution is fundamentally different from that of eGaIn, since it is the movement of hydrated ions that makes a saline solution electrically conductive.

\section{B. Electrically Conductive Interface}

Although the oxide skin of eGaIn [26], [31] possesses many characteristics that would make it a good electrode for an ionic solution (highly conductive and oxidation resistant), the mechanical properties of both liquids make direct contact between them infeasible, the viscosity of eGaIn is too low to provide a mechanical barrier against mixing of the two different liquids under external stress. One of the main challenges in this work was therefore to create a soft conductive interface between the saline solution and eGaIn. This soft interface must act as an electrode between the saline solution and the eGaInfilled microchannels. It should be highly conductive but with very low piezoresistivity and should not affect the mechanical properties of the sensor. For this purpose, we use a platinumcured silicone elastomer doped with conductive micro and nanoparticles.

Since the conductivity and the elasticity of the resulting composite have opposing dependencies on the amount of conductive particles, these two variables seemed irreconcilable. In order to minimize the loss of elasticity, we used nanoparticles with a high aspect ratio, since such geometry is known to provide good conductivity at lower filler volumes [32]. The particle chosen was a nickel nanostrand (NiNs, length $30 \mu \mathrm{m}$, Conductive Composites inc.). Nickel is highly conductive, affordable, slow to oxidize, and hence suitable for our application. The nanopowder was incorporated into a very soft silicone (EcoFlex, Smooth-On) of Shore hardness 00-30. To avoid damage to the strands, the resulting composite was mixed for three minutes using a planetary mixer (Thinky ARE-310). These high-aspect-ratio particles are known to form agglomerates in spite of powerful mixing [32]. In order to increase the overall electrical conductivity, we used a small amount of chopped nickel-coated carbon fiber (NiCCF, Conductive Composites inc.) to interconnect the nanostrand agglomerates [33]. Moreover, the Gallium contained in the eGaIn alloy, is known to corrode metals such as Nickel [34], and even though very few contact points are possible, the use of carbon based fibers thus further increases the reliability of the interfaces in terms of conductivity.

It is also well known that long particles result in good conductivity at low filler volumes [35]. However, embedding long particles into a soft silicone matrix is also known for creating mechanical hysteresis. Indeed, as pointed by Rogers et al. [36], the large differences in mechanical properties between soft silicone and long carbon fibers creates highly heterogeneous systems with a nonlinear behaviour in

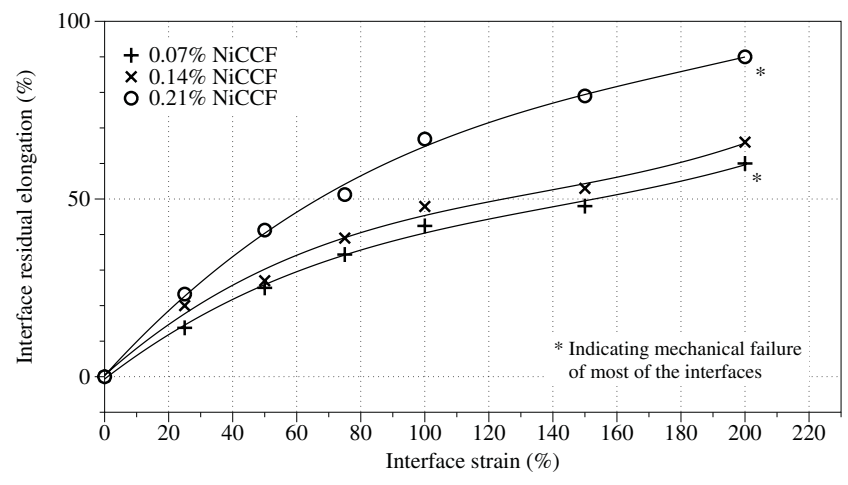

Fig. 3. Impact of the NiCCF on interfaces of fixed NiNs (4\% per vol.) aspect ratio.

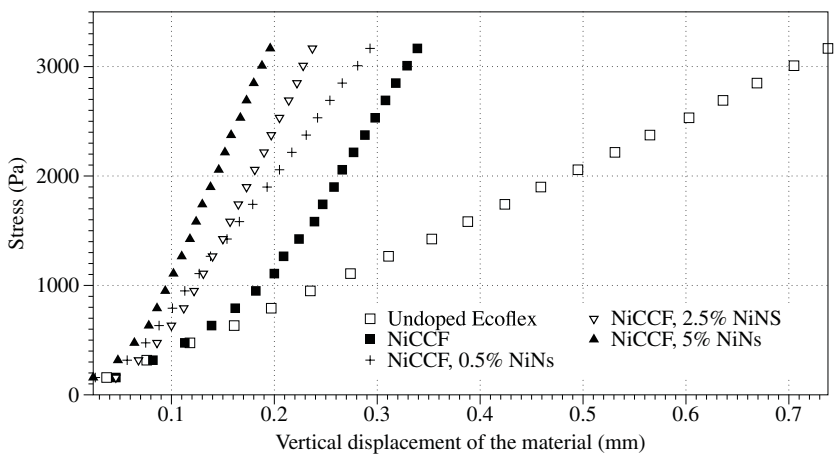

Fig. 4. Comparison of the mechanical response of doped (NiNs and $0.07 \%$ per vol. NiCCF) and non-doped EcoFlex.

buckling mode. Figure 3 illustrates the mechanical response of several interfaces made with different amounts of NiCCF. This figure shows the residual increase in length after stretch. We can observe that even for a very small amount of NiCCF ( $0.07 \%$ by volume), the residual length after stretch is considerable. Therefore, to minimize this effect we decided to use the smallest amount of NiCCF in our interface.

The NiNs particles being significantly smaller than NICCF particles, they do not contribute noticeably to mechanical hysteresis in the composite. However, the amount of NiNs that is required to form enough effective conductive pathways trough the silicone is higher than with NiCCF. Typically, increasing the volume ratio of filler in a composite will also increase the compressive strength of the resulting material [37]. Figure 4 shows how the compressive strength of our silicone composite increases with the amount of NiNs used. In order to keep the properties of our sensor as homogeneous as possible, conductive interfaces with a high compressive strength should be avoided. Hence, the final NiNs and NiCCF proportion used in our interfaces was chosen as a trade-off between the two above undesired effects: the chosen mixture contained $4 \%$ NiNs and $0.09 \%$ (length $800 \mu \mathrm{m}$ ) NiCCF by volume. The measured resistivity was $80 \Omega / \mathrm{cm}$, which was much greater than eGaIn, but negligible compared to that of the ionic solution [23]. The interaction between the two different particle types is apparent in the scanning electron microscopy image shown in Figure 5. 


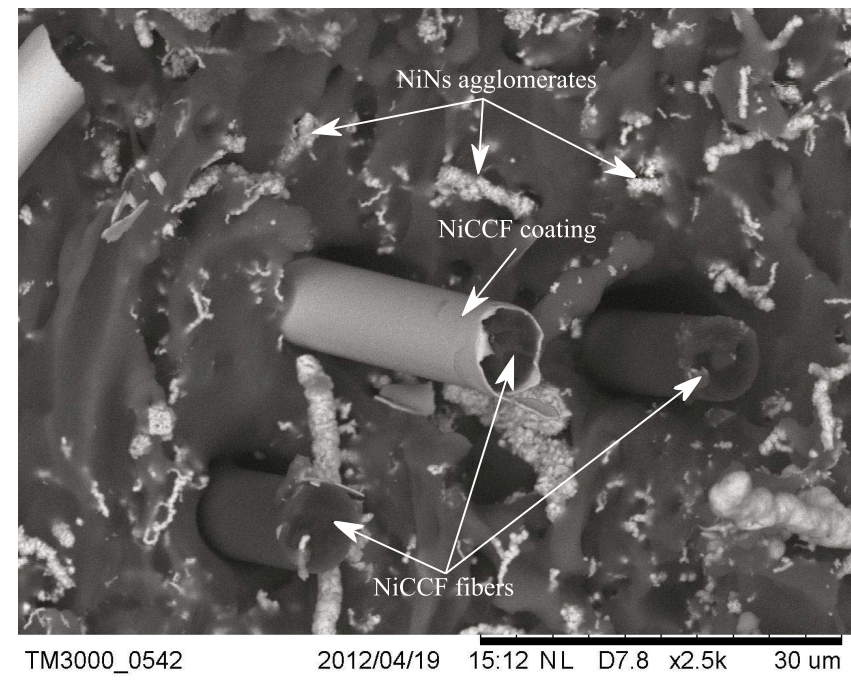

Fig. 5. Scanning electron microscope image at $2500 \mathrm{X}$ of the nanocomposite used for the conductive interfaces of the soft strain sensor.

\section{Electrical Interface}

At voltages above $1.23 \mathrm{~V}$, electrolysis of water divides molecules to produce hydrogen and oxygen gas. Thus, we designed a relaxation oscillator circuit using a low-voltage operational amplifier (TLV 2362, Texas Instruments) in order to obtain an integrated low-voltage AC circuit. This simple circuit measures the resistance change of the sensor and has the advantage of using a DC supply to generate a $-1 \mathrm{~V}$ to $1 \mathrm{~V}$ oscillation where the pulse length is a linear function of the sensor resistance, according to the following equation:

$$
T=2 R_{S} C_{1} \ln \frac{1+B}{1-B},
$$

where $R_{s}$ is the resistance of the sensor, $C_{1}$ is the oscillator capacitance, with sensor's capacitance being negligible in comparison, and $B$ is a function of resistances $R_{1}$ and $R_{2}$, specifically,

$$
B=\frac{R_{1}}{R_{1}+R_{2}} .
$$

As the sensor is stretched, the resistance of the ionic solution will increase and the frequency of the resulting oscillation will decrease. Previous characterization of the dependence of the impedance of the ionic solution on frequency [23], [38] shows that the frequency of the AC signal can be a major influence on the flow of ions and thus on the real part of the impedance. Direct current (or an extremely low frequency) will cause electrolysis and polarize the electrodes. At the other extreme, an extremely high frequency will increase the conductivity of the solution by the Debye-Falkenhagen effect [39]. As previously shown, at frequencies between $40 \mathrm{~Hz}$ and 10 $\mathrm{MHz}$, the real part of the impedance will slowly decrease in a predictable way [23]. The dependence of the resistance of the ionic solution on frequency will have an impact on eq. (1) as the relationship becomes coupled. Not only does resistance have an impact on oscillation frequency, oscillation frequency has an impact on resistance. Therefore, eq. (1) can be rewritten

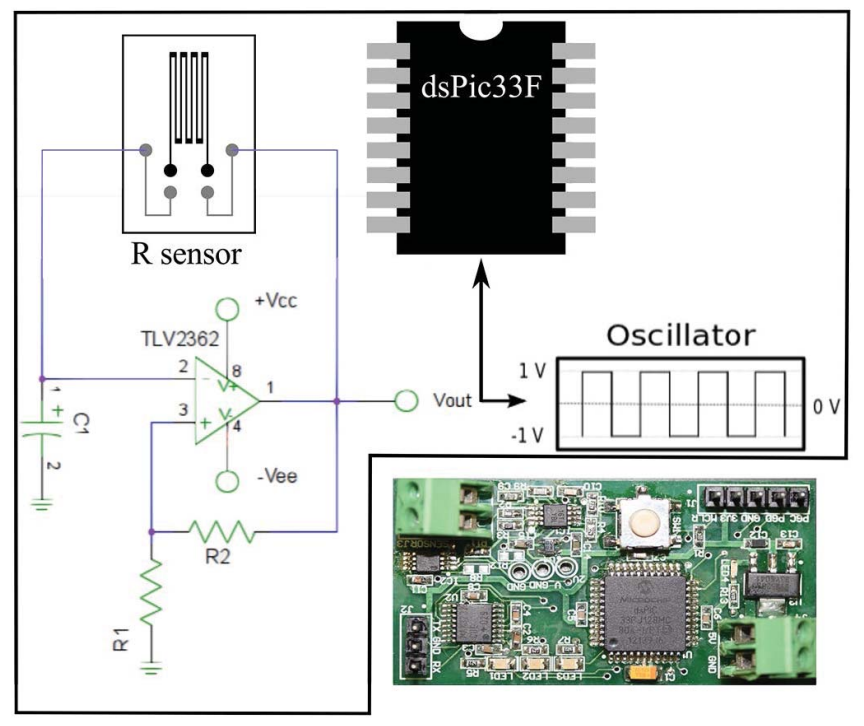

Fig. 6. Circuit designed for the soft strain sensor and the resulting PCB.

as follows:

$$
T=2 R_{S}(f) C_{1} \ln \frac{1+B}{1-B} .
$$

This equation is no longer linear, which has the effect of increasing the perceived gauge factor. To maximize this coupling effect, the components $\mathrm{C} 1, \mathrm{R} 1$ and $\mathrm{R} 2$ in the circuit were chosen such that the oscillation frequency at the sensor nominal resistance was around $600 \mathrm{~Hz}$, following the analysis of the data presented in [23], [38], [40]. The oscillation frequency is measured using a counter input of a digital signal processor (dsPIC33F, Microchip Technology). Figure 6 shows a schematic of the oscillator circuit and the completed printed circuit board (PCB).

\section{FABRICATION}

The sensor was fabricated according to the procedure illustrated in Figure 7. Three acrylic molds were engraved in Stucki mode ${ }^{2}$ using a $\mathrm{CO}_{2}$ laser cutter (Epilog Helix 40 Watts): one for casting the conductive interfaces, another for the sensor layer with microchannels and the last for the top layer. The laser allow us to make channel widths varying from 200 to $550 \mu \mathrm{m}$ (from top to bottom). By controlling the power and speed of the laser, channel depth could also be controlled. The present prototype is $2 \mathrm{~mm}$ thick with channel depth of $600 \mu \mathrm{m}$. Figure 8 shows a close-up view of the resulting mold with the microchannel pattern, including channel dimensions. This figure also shows that the laser produces non-square (trapezoidal) channel geometry.

As mentioned above, the base material of the conductive interface is a platinum cure silicone (EcoFlex, Smooth-On) with a very low Shore hardness (00-30). The conductive nano and micro particles, NiNs ( $4 \%$ by volume) and NiCCF $(0.09 \%$ by volume) were embedded into the elastomer by mixing for

\footnotetext{
${ }^{2}$ The Stucki algorithm is a graphical dithering technique, aiming at reducing the quantisation error by averaging each pixel's depth with the neighbouring pixels of the image. Laser engraving using this algorithm leads to a better surface finish [41].
} 
Step 1 : Making molds with the laser cutter
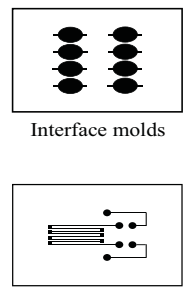

First layer mold

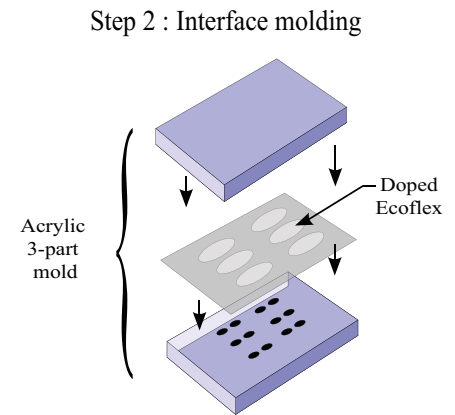

Step 3 : First layer molding and rastering
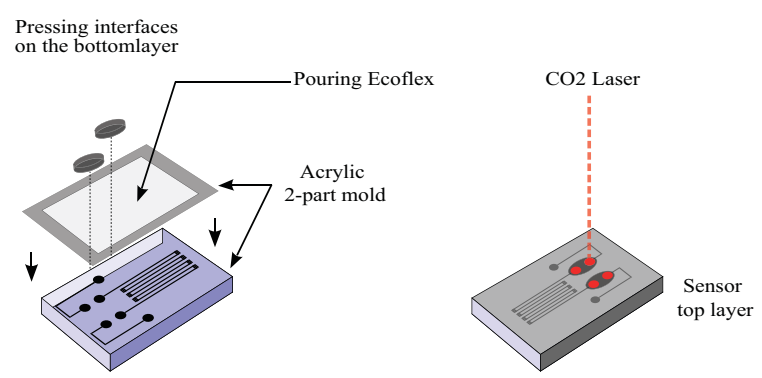

Step 4 : Bonding the layers

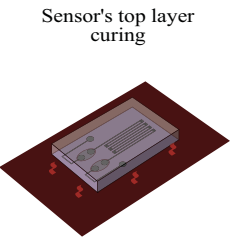

Heating first layer

Step 5 : Injecting fluids
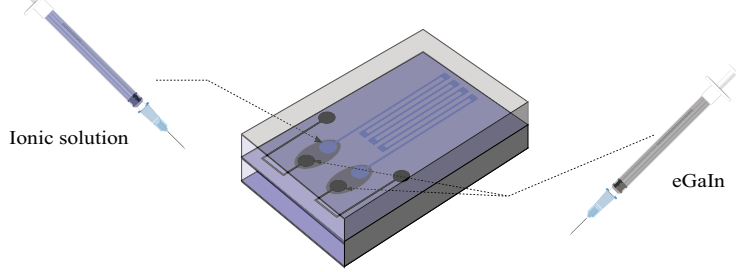

Fig. 7. Soft strain sensor fabrication and assembly procedure.

three minutes in the planetary mixer. The resulting composite was degassed in a vacuum chamber, poured into the clean mold, and cured for 25 minutes in an oven at $65^{\circ} \mathrm{C}$.

The cured conductive interfaces were placed in their positions in the sensor mold and additional EcoFlex was poured to form the portion of the sensor that contains the microchannels. After 30 minutes of curing at $65{ }^{\circ} \mathrm{C}$, the $\mathrm{CO}_{2}$ laser was used at low power (30\% of maximum speed, $22 \%$ of maximum power) to micro-machine the interfaces in order to remove the poorly conducting elastomer layer formed during curing and thereby expose the nanoparticles on the surfaces that will contact the fluids. This did not affect the nickel particles, since the laser wavelength $(10.6 \mu \mathrm{m})$ was too long to be absorbed



Fig. 8. Acrylic mold after laser engraving in Stucki mode and its dimensions.

by this metal [42]. The resistance of the resulting interface was as low as $30 \Omega$ throughout its entire length $(4 \mathrm{~mm})$. It was found that the resistance could be decreased by at least one order of magnitude by micro-machining the top of the interfaces, and that repeatability was increased as a result of different interfaces having much closer nominal resistances. Figure 9 shows the interface material before and after laser treatment.

The top layer that covers the microchannels was then cast and bonded to the cured bottom layer. Since silicone elastomer is extremely hydrophobic, bonding two layers can be difficult. Although oxygen plasma bonding [43] is often used to bond Polydimethylsiloxane (PDMS), attempts to bond Ecoflex using this method were unsuccessful. To achieve good bonding, the patterned layer was kept in the oven at $65{ }^{\circ} \mathrm{C}$ while the top layer was spin-coated with Ecoflex at low speed $(350 \mathrm{rpm})$, and then for the last 20 seconds at a greater speed $(1600 \mathrm{rpm})$ spreading the Ecoflex as a flat, uniform and very thin layer, thus preventing the channels to be filled in the next step, as shown in previous work [16]. The heated layer was then carefully placed on the spin-coated layer, and the assembly was deposited in the oven for 15 minutes at $65^{\circ} \mathrm{C}$.

The final steps consisted of filling the microchannels with eGaIn and saline solution using syringes fitted with a hypodermic needle (one for injecting the liquid and another for withdrawing captured air at the other end of the channel). Small wires are inserted at the end of the eGaIn channels. Ecoflex is then spread in small amounts around the connection point, thus sealing the holes left by the needles and attaching the wires that link the sensor to the PCB. The sensor is finally cured at room temperature.

\section{Results}

In order to test the robustness of our design, in particular the effectiveness of our conductive interface at eliminating electrode polarization and electrolysis, the sensor was first connected to the circuit shown in Figure 6 and stimulated with AC current for over 100 hours. No bubble formation or change in resistance was observed in the sensor. In comparison, a single hour under 3V DC was sufficient to cause bubble 


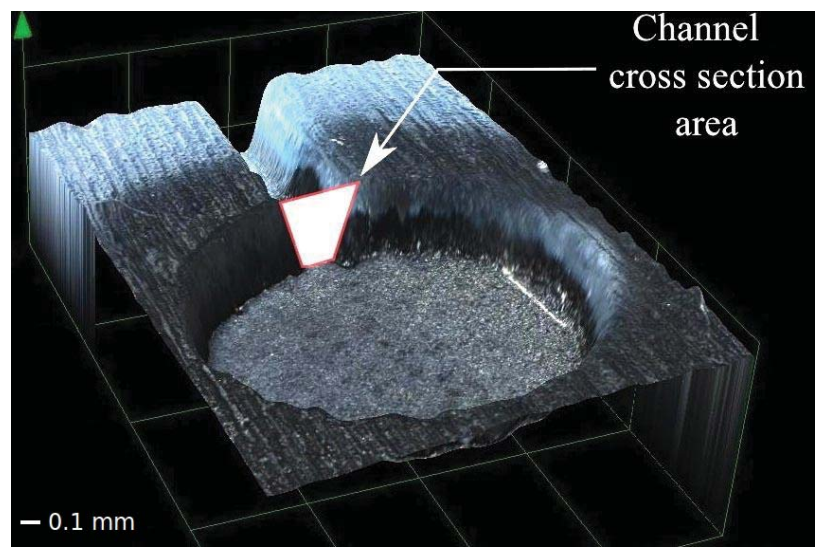

(a)

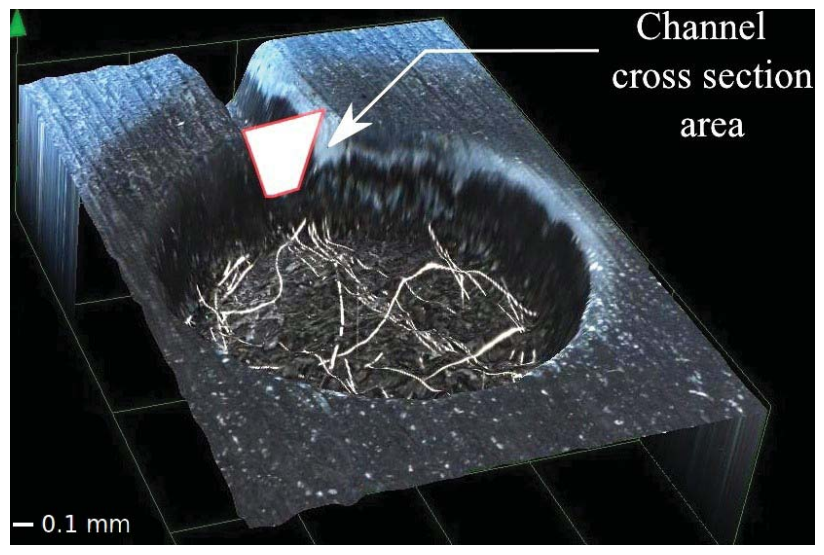

(b)

Fig. 9. 3D views of the sensor composite interface taken with an opto-digital microscope: (a) before micro-machining and (b) after micro-machining.

formation in an earlier prototype and render the device nonconductive.

However, after long term storage at room temperature of the sensor (weeks), some evaporation of the water contained in the ionic solution was noted. This change created a proportional small drift of the resistance of the ionic solution channel, although it did not change the overall behaviour of the sensor. There could be many sources for this evaporation such as an imperfect bonding between the sensor layers or an imperfect closure of the syringe's holes. Although we didn't find any information regarding the gas permeability of Ecoflex, the permeability of this silicone is another source that could be responsible for these losses. Ionic Liquids have been proposed in comparable situation to overcome this effect as they are both conductive and have very low volatility [24], [44], [45] and are investigated for further stability of the sensor.

Stress was also applied to the eGaIn channels to verify that the sensor was sensitive only to stretching of the portion containing the saline-solution-filled microchannels. Neither stretching nor pressure applied to the eGaIn channels produced any significant change in the overall sensor resistance, up to the point at which these channels were pinched and rendered non conductive.

Following these qualitative experiments, the sensor was mounted on a force test stand (Mark-10 ES20,) equipped with a force and a displacement gauge (Mark-10 M4-10 and Mitutoyo 543-693, respectively) to measure the relationship between resistance and strain (see Figure 10). The resistance variation of the sensor was monitored with a LCR meter (B\&K Precision 878B) at a frequency of $1 \mathrm{KHz}$. The purpose of this test was to characterize the sensor response and verify the gauge factor as well as compare it to a theoretical model.

To model the change in the resistance of our sensor under strain, we had to consider the resistance of the solution as well as the effect of the geometry. The electrical resistance $R$ of a channel filled with an ionic solution is known to follow the relationship defined by the following equation:

$$
R=\rho \frac{L}{A},
$$

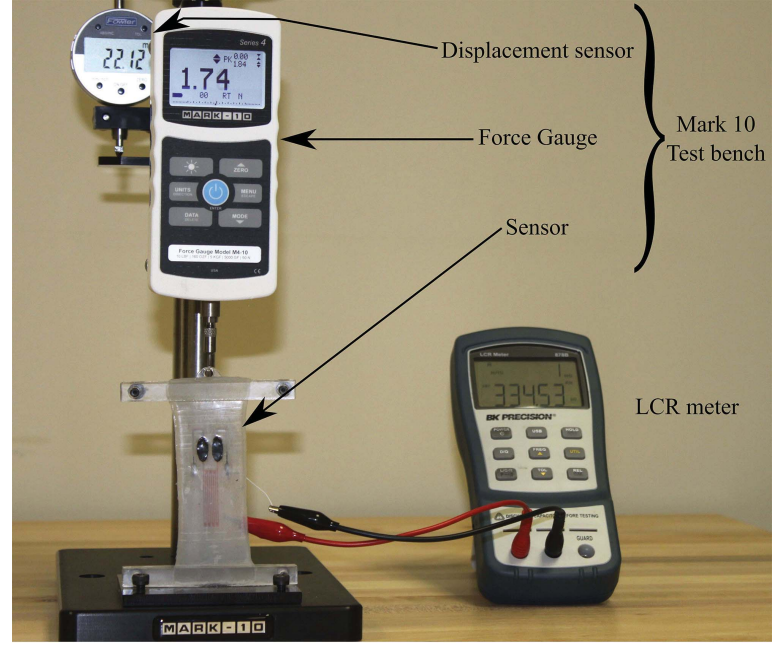

Fig. 10. Test setup used for the measurements made on the soft strain sensor.

where $\rho$ is the resistivity of the ionic solution, $A$ the smallest cross-sectional area of the channel, and $L$ is the length of the microchannel. Assuming incompressibility, the ionic solution can be modeled as a liquid with constant specific resistance $\rho$. The value of $\rho$ was calculated using the measured initial sensor dimensions ( $L$ and $A$ ) and the initial resistance at $1 \mathrm{KHz}$.

The specific resistance being constant, the variation in the resistance of the sensor is due solely to the change in the dimension $L$ and the cross-sectional area $A$. As shown in Figure 9, the channel cross-section is trapezoidal. The area of an isosceles trapezoid is calculated using the following equation:

$$
A=h \frac{a+b}{2},
$$

where $h$ is the trapezoid height, and $a$ and $b$ are, respectively, the top and bottom widths of the channels. Measurements made with an opto-digital microscope (Olympus DSX100) show that the channel initial height $h_{0}$ was $0.6 \mathrm{~mm}$ and that the maximal and minimal initial widths were $a_{0}=0.55 \mathrm{~mm}$ and $b_{0}=0.2 \mathrm{~mm}$, respectively. The initial channel length $L_{0}$ was designed to be $160 \mathrm{~mm}$. 


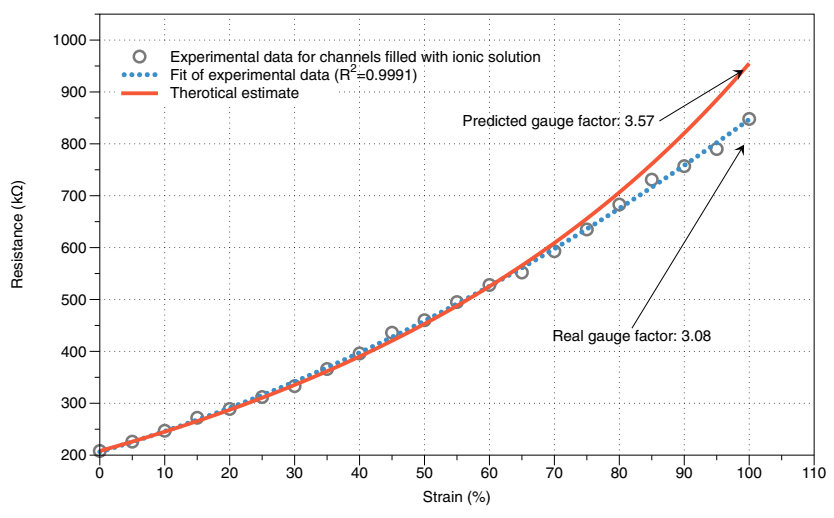

Fig. 11. Variation of the resistance as a function of strain for the ionic solution strain sensor.

The length of the sensor channels subject to a strain $\epsilon=\frac{\Delta L}{L_{0}}$ is expressed as follows:

$$
L=L_{0}+\Delta L=L_{0}(1+\epsilon) .
$$

In response to this stretching, the cross-sectional area $A$ of a channel will decrease as follows:

$$
A=\left(h_{0}+\Delta h\right) \frac{\left\{\left(a_{0}+\Delta a\right)+\left(b_{0}+\Delta b\right)\right\}}{2} .
$$

Using the relation defining transversal and axial strains, $\Delta h$, $\Delta a$ and $\Delta b$ can be, replaced, respectively by $-v \epsilon h,-v \epsilon a$ and $-v \epsilon b$, where $v$ is the Poisson's ratio of the material. By substituting these expressions into eq. (7), the relationship between the applied strain and the channel cross-sectional area can be expressed as follows:

$$
A=\frac{h_{0}\left(a_{0}+b_{0}\right)(1-v \epsilon)^{2}}{2} .
$$

Substituting eqs. (6) and (8) into eq. (4), the resistance of the sensor for applied strain becomes

$$
R_{s}=\frac{2 L_{0} \rho(1+\epsilon)}{h_{0}\left(a_{0}+b_{0}\right)(1-v \epsilon)^{2}} .
$$

It should be noted that the mechanical property of the resulting sensor is anisotropic due to the presence of the channels and the fluids and that the conventional value of 0.5 for the Poisson's ratio of an elastomeric material is therefore not applicable. This ratio was instead calculated using experimental measurements of the cross sectional area at different strains under a microscope. The Poisson's ratio $v$ determined experimentally for our sensor was 0.39 .

\section{A. Sensor Resistance Variation Under Strain}

Figure 11 shows the measured resistance as a function of strain as well as the theoretical curve obtained with the model. It is noted that for strain less than $70 \%$, the model appears to match the experimental data almost perfectly. The less pronounced slope of the experimental curve for the portion above $70 \%$ leads to a smaller gauge factor (3.08) than what was anticipated based on the model (3.57).

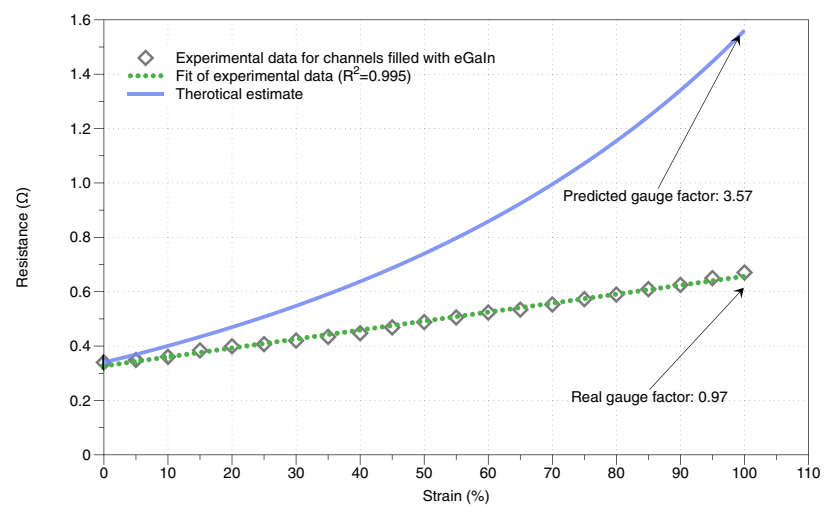

Fig. 12. Variation of the resistance as a function of strain for the eGaIn sensor.

\section{B. Comparaison With eGaIn}

Although the main motivation for the hybrid (ionic solution/eGaIn) approach was to decouple the sensing portion from the signal routing channels in order to make the sensor less sensitive to stress on the signal-carrying portions, we also wished to verify the impact of replacing eGaIn with saline solution on the sensor response. We therefore filled a sensor entirely with eGaIn and measured its strain response using the same test setup. Figure 12 shows the measured resistance of the eGaIn sensor, as well as the theoretical curve given by the model represented by eq. (9). The experimental curve differs considerably from the theoretical prediction. In fact, instead of the expected nearly quadratic function, we obtained a quasilinear relation that follows the change in channel length quite precisely:

$$
R_{S} \approx k L_{0}(1+\epsilon),
$$

where $k$ is a constant defined as $\frac{\rho}{A_{0}}$. This has the effect of lowering the gauge factor of the eGaIn-filled sensor to a considerable extent ( 0.97 instead of 3.57 theoretically at $\epsilon=1)$.

Figure 13 shows the measured normalized change in resistance versus applied strain for the ionic solution sensor and for the the eGaIn only sensor. It is noted that the gauge factor is more than three times (3.17 times) greater for the ionic solution sensor.

The gauge factor $G$ of a sensor for a given temperature is given by $G=\Delta R / R_{0} \epsilon$, where $\Delta R$ is the sensor change of resistance, $R_{0}$ is initial resistance and $\epsilon$ the strain. Using eqs. (9) and (4), the gauge factors of our sensor is :

$$
G=\frac{1+\epsilon-(1-v \epsilon)^{2}}{\epsilon(1-v \epsilon)^{2}} .
$$

According to this equation, the gauge factor is a function only of the strain and the Poisson's ratio and therefore should be the same no matter what conductive fluid is used.

Our hypothesis to explain this discrepancy is that the surface tension of the oxidized skin of eGaIn prevents this nonNewtonian liquid from forming the exact shape of the channel. This surface tension is known to be very high [26]. Therefore, up to a certain critical stress and channel cross-sectional area, it is possible that the effective area of the liquid therefore remains constant. Observations made at higher strains than 


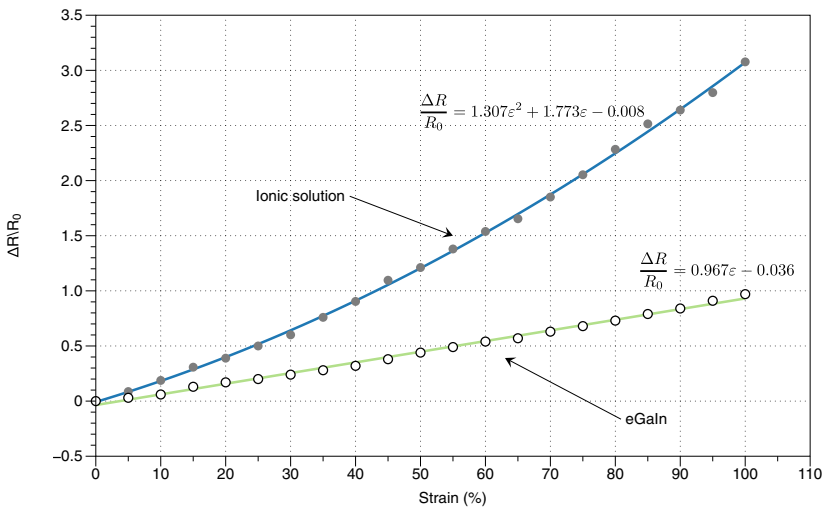

Fig. 13. Normalized change in resistance as a function of strain for salinefilled and eGaIn-filled soft strain sensors.

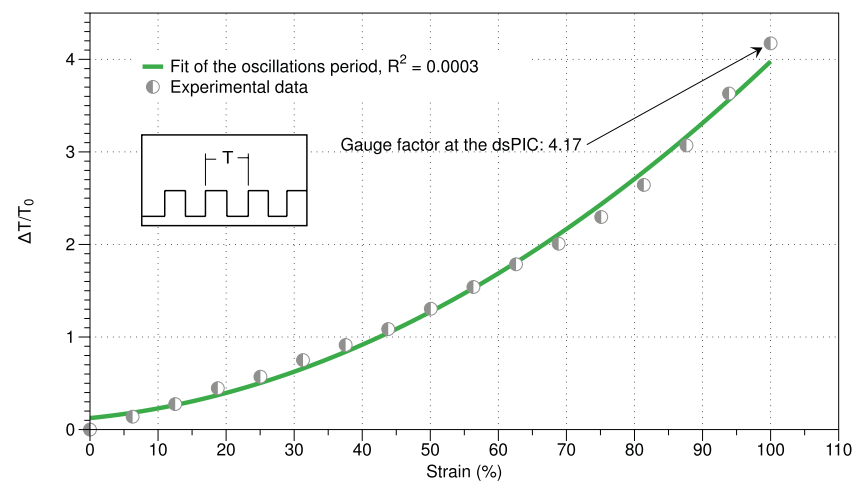

Fig. 14. Normalized change in the oscillation period as a function of strain for the sensor filled with ionic solution.

presented in these graphs support this explanation. Beyond a strain of $130 \%$, the curve loses its linearity and acquires the characteristic of a quadratic system in which the channel length and the cross-sectional area both change.

\section{Frequency Dependency of the Sensor Impedance}

One other point to validate was the frequency dependency of the sensor impedance on the perceived gauge factor at the micro-controller. As mentioned in section II-C, the resistance of an ionic solution is a function of the frequency of the $\mathrm{AC}$ input at the electrodes. The circuit used to power the sensor and measure its response is a low-voltage op-amp that oscillates at a frequency that is a function of sensor resistance, based on eq. (3). This generates coupling that will increase the perceived gauge factor. To validate this hypothesis, the sensor filled with ionic solution was tested using the apparatus shown in Figure 10 but connected this time to the circuit shown in Figure 6. Since the resistance will be measured in terms of the duration of an oscillation rather than by reading a voltage we recorded the normalized change in the period of the oscillation as a function of the strain applied to the sensor in order to calculate the gauge factor at the microcontroller (see Figure 14). As anticipated, the coupling in our circuit increased the gauge factor of the sensor (from 3.08 to 4.17) compared to when its resistance was measured at a fixed frequency.

\section{Sensor Stiffness}

Finally, since our sensor was designed for the purpose of measuring motion associated with the phalanges of a

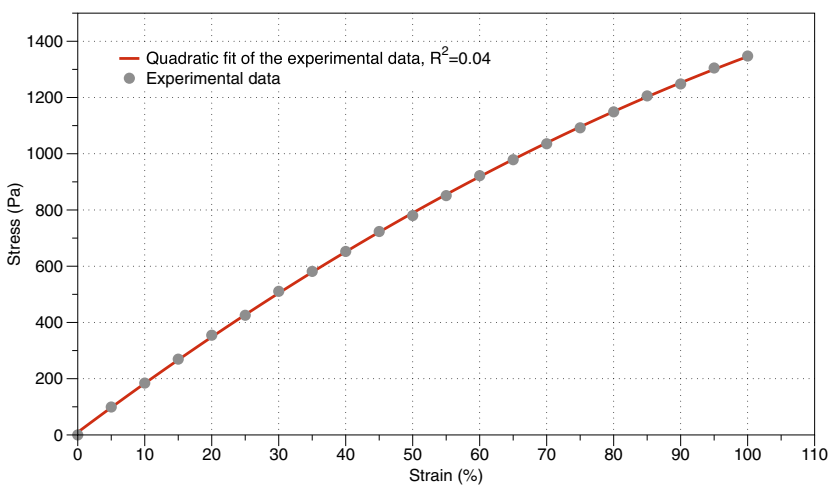

Fig. 15. The stress-strain relationship of the soft strain sensor.

proprioceptive glove, the stiffness of the material should not interfere with the movement of the prosthetic hand. Indeed, a high stiffness could impede the grasp motion of the prosthetic hand. Figure 15 shows the strain versus pulling force response of the sensor. Although the stiffness appears non-linear, it is sufficiently predictable and low enough to allow stretching without significant interference with our application. Preliminary tests suggested that in the context of our application the maximum strain that our sensor will undergo is around $\epsilon=10 \%$. According to Figure 15, reaching this strain required a pulling force of few hundred millinewton, which is significantly below typical prehension forces [46].

\section{CONCLUSION}

This paper proposes a novel hybrid soft elastomer strain sensor in which signal routing is decoupled from sensing in order to make the sensor insensitive to undesired stress applied to the wiring. To achieve this goal, we used two conductive liquids of differing resistivity, namely a saline solution and eGaIn.

One of the challenges of this hybrid approach was to build soft but conductive interfaces between the two different liquids. By using a mixture of high-aspect-ratio nickel micro/nanoparticles, we obtained good conductivity without affecting the mechanical properties of the interface elastomer matrix. We also designed a self-oscillating low-voltage circuit that can easily power the sensor as well as provide a measurement of its resistance change.

Several tests were performed in order to characterize the sensor. The results showed that the proposed sensor could be modeled accurately by considering the conductivity of the solution and the change in geometry. The results also showed that the effective gauge factor of the hybrid sensor was greater than that of a sensor filled only with eGaIn. We also demonstrated that by exploiting the dependency of the resistance of the ionic solution on the frequency of the applied voltage, we could increase the gauge factor measured at the micro-controller.

We believe that by incorporating different fluids into the same stretchable soft sensor, this work opens avenues to further integration of microfluidics into elastomer-based devices. Future work will be oriented toward interfacing the end of the eGaIn channels with a flexible circuit as well 
as building a full-scale prototype of a flexible proprioceptive glove.

\section{REFERENCES}

[1] V. Feliu and F. Ramos, "Strain gauge based control of single-link flexible very lightweight robots robust to payload changes," Mechatronics, vol. 15 , no. 5 , pp. 547-571, Jun. 2005.

[2] J. Butterfaß, M. Grebenstein, H. Liu, and G. Hirzinger, "DLR-hand II: Next generation of a dextrous robot hand," in Proc. Proc. IEEE Int. Conf. Robot. Autom., vol. 1. May 2001, pp. 109-114.

[3] M. Carrozza, A. Eisinberg, A. Menciassi, D. Campolo, S. Micera, and P. Dario, "Towards a force-controlled microgripper for assembling biomedical microdevices," J. Micromech. Microeng., vol. 10, no. 2 , p. 271, Jun. 2000

[4] F. Suganuma, A. Shimamoto, and K. Tanaka, "Development of a differential optical-fiber displacement sensor," Appl. Opt., vol. 38, no. 7, pp. 1103-1109, Mar. 1999.

[5] S. Kiesel, K. Peters, T. Hassan, and M. Kowalsky, "Behaviour of intrinsic polymer optical fibre sensor for large-strain applications," Meas. Sci. Technol., vol. 18, no. 10, p. 3144, Oct. 2007.

[6] Y.-L. Park, S. C. Ryu, R. J. Black, K. K. Chau, B. Moslehi, and M. R. Cutkosky, "Exoskeletal force-sensing end-effectors with embedded optical fiber-bragg-grating sensors," IEEE Trans. Robot., vol. 25, no. 6, pp. 1319-1331, Dec. 2009.

[7] M. Dobrzynski, R. Pericet-Camara, and D. Floreano, "Contactless deflection sensor for soft robots," in Proc. IEEE/RSJ Int. Conf. Intell. Robot. Syst., Jul. 2011, pp. 1913-1918.

[8] M. Knite, V. Teteris, A. Kiploka, and J. Kaupuzs, "Polyisoprene-carbon black nanocomposites as tensile strain and pressure sensor materials," Sens. Actuators A, Phys., vol. 110, nos. 1-3, pp. 142-149, Feb. 2004.

[9] I. Kang, M. Schulz, J. Kim, V. Shanov, and D. Shi, "A carbon nanotube strain sensor for structural health monitoring," Smart Mater. Struct., vol. 15, no. 3, p. 737, Jun. 2006.

[10] O. Johnson, G. Kaschner, T. Mason, D. Fullwood, T. Hyatt, B. Adams, K. Cole, and G. Hansen, "Extreme piezoresistivity of silicone/nickel nanocomposites for high resolution large strain measurement," in Proc. TMS Ann. Meeting, 2010, pp. 2197-2206.

[11] K. Loh, J. Lynch, B. Shim, and N. Kotov, "Tailoring piezoresistive sensitivity of multilayer carbon nanotube composite strain sensors," J. Intell. Mater. Syst. Struct., vol. 19, no. 7, pp. 747-764, Jul. 2008.

[12] M. Lacasse, V. Duchaine, and C. Gosselin, "Characterization of the electrical resistance of carbon-black-filled silicone: Application to a flexible and stretchable robot skin," in Proc. IEEE Int. Conf. Robot. Autom., May 2010, pp. 4842-4848.

[13] N. Hu, Y. Karube, C. Yan, Z. Masuda, and H. Fukunaga, "Tunneling effect in a polymer/carbon nanotube nanocomposite strain sensor," Acta Mater, vol. 56, no. 13, pp. 2929-2936, Aug. 2008.

[14] O. Johnson, C. Gardner, D. Fullwood, B. Adams, N. Hansen, and G. Hansen, "The colossal piezoresistive effect in nickel nanostrand polymer composites and a quantum tunneling model," Comput., Mater., Continua, vol. 15, no. 2, pp. 87-112, 2010.

[15] Y.-L. Park, C. Majidi, R. Kramer, P. Bérard, and R. J. Wood, "Hyperelastic pressure sensing with a liquid embedded elstomer," J. Micromech. Microeng., vol. 20, no. 12, p. 125029, Dec. 2010.

[16] Y.-L. Park, B. Chen, and R. J. Wood, "Design and fabrication of soft artificial skin using embedded microchannels and liquid conductors," IEEE Sensors J., vol. 12, no. 8, pp. 2711-2718, Aug. 2012.

[17] R. Kramer, C. Majidi, R. Sahai, and R. J. Wood, "Soft curvature sensors for joint angle proprioception," in Proc. IEEE/RSJ Int. Conf. Intell. Robot. Syst., Sep. 2011, pp. 1919-1926.

[18] C. Majidi, R. K. Kramer, and R. J. Wood, "A non-differential elastomer curvature sensor for softer-than-skin electronics," Smart Mater. Struct., vol. 20, no. 10, p. 105017, Oct. 2011.

[19] T. Lalibert, M. Baril, F. Guay, and C. Gosselin, "Towards the design of a prosthetic underactuated hand," in Proc. IFToMM/ASME Int. Workshop Underactuated Grasping, Aug. 2010, pp. 19-26.

[20] M. Baril, T. Laliberté, F. Guay, and C. Gosselin, "Static analysis of single-input/multiple-output tendon-driven underactuated mechanisms for robotic hands," in Proc. Int. Design Eng. Tech. Conf. Comput. Inf. Eng. Conf., Aug. 2010, pp. 1-10.

[21] M. Carrozza, G. Cappiello, E. Cavallaro, S. Micera, F. Vecchi, and P. Dario, "Design and control of an underactuated cybernetic artificial hand," in Proc. World Autom. Congr., vol. 15. Jul. 2004, pp. 111-116,

[22] A. Blank, A. Okamura, and K. Kuchenbecker, "Identifying the role of proprioception in upper-limb prosthesis control: Studies on targeted motion," ACM Trans. Appl. Perception, vol. 7, no. 3, pp. 1-15, Jun. 2010.
[23] Y.-N. Cheung, Y. Zhu, C.-H. Cheng, C. Chao, and W. W.-F. Leung, "A novel fluidic strain sensor for large strain measurement," Sens. Actuators A, Phys., vol. 147, no. 2, pp. 401-408, Oct. 2008.

[24] C.-Y. Wu, W.-H. Liao, and Y.-C. Tung, "Integrated ionic liquid-based electrofluidic circuits for pressure sensing within polydimethylsiloxane microfluidic systems," Lab on a Chip, vol. 11, pp. 1740-1746, May 2011.

[25] T. Liu, P. Sen, and C. Kim, "Characterization of liquid-metal galinstan for droplet applications," in Proc. IEEE 23rd Int. Conf. Micro Electro Mech. Syst., Jan. 2010, pp. 560-563.

[26] M. Dickey, R. Chiechi, R. Larsen, E. Weiss, D. Weitz, and G. Whitesides, "Eutectic gallium-indium (eGaIn): A liquid metal alloy for the formation of stable structures in microchannels at room temperature," Adv. Funct. Mater., vol. 18, no. 7, pp. 1097-1104, 2008.

[27] W. Irshad and D. Peroulis, "A silicon-based galinstan magnetohydrodynamic pump," in Proc. 9th Int. Workshop Micro Nanotechnol. Power Generat. Energy Convers. Appl., 2009, pp. 127-129.

[28] K. Shaikh and C. Liu, "A bi-stable latchable pdms valve employing low melting temperature metal alloys," in Proc. IEEE Trans. Solid-State Sensors, Actuat. Microsyst. Conf., Jun. 2007, pp. 2199-2202.

[29] D. Lipomi, B. Tee, M. Vosgueritchian, and Z. Bao, "Stretchable organic solar cells," Adv. Mater, vol. 23, no. 15, pp. 1771-1775, Apr. 2011.

[30] S. Cheng and Z. Wu, "A microfluidic, reversibly stretchable, largearea wireless strain sensor," Adv. Funct. Mater, vol. 21, no. 12, pp. 2282-2290, Jun. 2011.

[31] Q. Xu, N. Qudalov, Q. Guo, H. Jaeger, and E. Brown, "Effect of oxidation on the mechanical properties of liquid gallium and eutectic gallium-indium," Phys. Fluids, vol. 24, no. 6, pp. 063101-1-063101-13, Jun. 2012.

[32] J. Baldyga, W. Orciuch, L. Makowski, K. Malik, G. Özcan-Taskin, W. Eagles, and G. Padron, "Dispersion of nanoparticle clusters in a rotor-stator mixer," Ind. Eng. Chem. Res., vol. 47, no. 10, pp. 3652-3663, Jan. 2008.

[33] H. Chung, S. Jang, H. Ryu, and K. Shim, "Effects of nano-carbon webs on the electrochemical properties in $\mathrm{LiFePO}_{4} / \mathrm{C}$ composite," Solid State Commun., vol. 131, no. 8, pp. 549-554, Aug. 2004.

[34] P. Luebbers and O. Chopra, "Compatibility of iter candidate materials with static gallium," in Proc. 16th IEEE/NPSS Symp. Fusion Eng., vol. 1. Oct. 1995, pp. 232-235.

[35] P. Lee, J. Lee, H. Lee, J. Yeo, S. Hong, K. H. Nam, D. Lee, S. S. Lee, and S. H. Ko, "Highly stretchable and highly conductive metal electrode by very long metal nanowire percolation network," Adv. Mater., vol. 24, no. 25, pp. 3326-3332, Jul. 2012.

[36] J. A. Rogers, T. Someya, and Y. Huang, "Materials and mechanics for stretchable electronics," Science, vol. 327, no. 5973, pp. 1603-1607, Mar. 2010.

[37] K. Fang, C. Weng, and S. Ju, "An investigation into the mechanical properties of silicon nanoparticles using molecular dynamics simulations with parallel computing," J. Nanoparticle Res., vol. 11, no. 3, pp. 581-588, Apr. 2009.

[38] E. McAdams, A. Lackermeier, J. McLaughlin, D. Macken, and J. Jossinet, "The linear and non-linear electrical properties of the electrode-electrolyte interface," Biosensors Bioelectron., vol. 10, nos. 1-2, pp. 67-74, 1995.

[39] F. Harris and C. O'Konski, "Dielectric properties of aqueous ionic solutions at microwave frequencies," J. Phys. Chem., vol. 61, no. 3, pp. 310-319, Mar. 1957.

[40] E. Ferrara, L. Callegaro, and F. Durbiano, "Optimal frequency range for the measurement of A.C. Conductivity in aqueous solutions,' in Proc. 17th IEEE Instrum. Meas. Technol. Conf., vol. 2. May 2000, pp. $775-779$.

[41] P. Stucki, "Algorithms and procedures for digital halftone generation," in Proc. Int. Soc. Opt. Photon. Symp. Electron. Imaging, Sci. Technol., May 1992, pp. 26-40.

[42] X. Chen, W. Lotshaw, A. Ortiz, P. Staver, C. Erikson, M. McLaughlin, and T. Rockstroh, "Laser drilling of advanced materials: Effects of peak power, pulse format, and wavelength," J. Laser Appl., vol. 8, no. 5, pp. 233-240, Jun. 1996.

[43] O. Zucker, W. Langheinrich, M. Kulozik, and H. Goebel, "Application of oxygen plasma processing to silicon direct bonding," Sens. Actuators A, Phys., vol. 36, no. 3, pp. 227-231, May 1993.

[44] Y. Zhu, C. Chao, C.-H. Cheng, and W.-F. Leung, "A novel ionic-liquid strain sensor for large-strain applications," IEEE Electron Device Lett., vol. 30, no. 4, pp. 337-339, Apr. 2009. 
[45] K. Noda, E. Iwase, K. Matsumoto, and I. Shimoyama, "Stretchable liquid tactile sensor for robot-joints," in Proc. IEEE Int. Conf. Robot. Autom., May 2010, pp. 4212-4217.

[46] J. Dargahi and S. Najarian, "Human tactile perception as a standard for artificial tactile sensinga review," Int. J. Med. Robot. Comput. Assist. Surgery, vol. 1, no. 1, pp. 23-35, Jun. 2005.

Jean-Baptiste Chossat is currently pursuing the Ph.D. degree at École de Technologie Supérieure, Montreal, QC, Canada. His current research interests include wearable computing, smart materials for sensors and actuators, and human-robot interaction.

Yong-Lae Park (M'10) received the M.S. and Ph.D. degrees in mechanical engineering from Stanford University, Stanford, CA, USA, in 2005 and 2010, respectively. He is currently a Technology Development Fellow with the Wyss Institute for Biologically Inspired Engineering, Harvard University, Boston, MA, USA. His current research interests include fiber optic force and tactile sensing, design of soft wearable robots for human rehabilitation and artificial skin with embedded soft sensors and actuators, and development of novel manufacturing processes for microrobots and 3-D smart-robot-structures.
Robert J. Wood (M'01) received the M.S. and Ph.D. degrees from the Department of Electrical Engineering and Computer Sciences, University of California, Berkeley, CA, USA, in 2001 and 2004, respectively. He is currently an Associate Professor with the School of Engineering and Applied Sciences and the Wyss Institute for Biologically Inspired Engineering, Harvard University, Boston, MA, USA. His current research interests include microrobotics and bioinspired robotics.

Vincent Duchaine received the B.Eng. and Ph.D. degrees in mechanical engineering from Université Laval, Québec, QC, Canada, in 2005 and 2010, respectively. He joined the Biomimetics Dexterous Manipulation Laboratory, Stanford University, Stanford, CA, USA, as a Post-Doctoral Fellow. He is one of the co-founders of Robotiq, Inc., a Canadian company that designs and manufactures flexible robotic grippers. He is currently a Professor with the Department of Automated Manufacturing Engineering, École de Technologie Supérieure, Montreal, QC, Canada. His current research interests include control and sensor design for improving intuitiveness and safety of physical human-robot interaction. 